\title{
Green-function Method for Nonlinear Interactions of Elastic Waves
}

\author{
Andriejus Demčenko \\ Division of Biomedical Engineering \\ University of Glasgow \\ Glasgow, UK \\ andriejus.demcenko@glasgow.ac.uk
}

\author{
Michael Mazilu \\ SUPA, School of Physics and Astronomy \\ University of St Andrews \\ St Andrews, UK \\ mm17@st-andrews.ac.uk \\ Jonathan M. Cooper \\ Division of Biomedical Engineering \\ University of Glasgow \\ Glasgow, UK \\ jon.cooper@glasgow.ac.uk
}

\author{
Julien Reboud \\ Division of Biomedical Engineering \\ University of Glasgow \\ Glasgow, UK \\ julien.reboud@glasgow.ac.uk
}

\begin{abstract}
In the linear wave propagation regime, an analytical mesh-free Green-function decomposition has been shown as a viable alternative to FDTD and FEM. However, its expansion into nonlinear regimes has remained elusive due to the inherent linear properties of the Green-function approach. This work presents a novel frequency-domain Green function method to describe and model nonlinear wave interactions in isotropic hyperelastic media. As an example of the capabilities of the method, we detail the generation of sum frequency waves when initial quasimonochromatic waves are emitted in a fluid by finite sources. The method is supported by both numerical and experimental results using immersion ultrasonic techniques.
\end{abstract}

Index Terms-Green functions, nonlinear ultrasonics, wave mixing

\section{INTRODUCTION}

Nonlinear ultrasonic measurements are attractive for industrial nondestructive testing in various engineering structures due to their higher sensitivity to changes of material properties than in linear cases. However, due to the limited availability of models of the nonlinear wave scattering in solids, which are often challenging to implement and resource-intensive, these measurements are not widely used in the industry. Most of the developed methods consider the linear wave propagation only, so there is a huge need for an efficient modeling technique for the wave scattering in the nonlinear regime. This will open up new measurement techniques with increased performance.

Numerical methods such as finite element [1], [2] and finite difference time domain [3] are used to model nonlinear wave interactions in 2D and 3D configurations. These well known methods become inefficient at higher frequencies ( $\mathrm{MHz}$ range and above) due to the requirement of 20 or more points per wavelength in the meshing grid. To overcome this constraint, we present a semi-analytical mesh-free method, based on Green functions, able to describe 3D elastic wave scattering in the nonlinear regime. The method enables us to study classical

This work was supported in part by the Engineering and Physical Sciences Research Council Fellowship under Grant EP/K027611/1 and in part by the European Research Council advanced investigator award under Grant 340117. and non-classical second order elastic waves interactions in isotropic hyperelastic media [4], [5].

\section{GREEN FUNCTION METHOD FOR NONLINEAR} INTERACTIONS OF ELASTIC WAVES IN ISOTROPIC MEDIA

A frequency domain Green tensor $G_{j i}\left(x_{k}-x_{0 k}\right)$ satisfies the following Navier-form equation of motion of a homogeneous isotropic hyperelastic solid

$$
\begin{array}{r}
\rho \mathrm{c}_{L}^{2} G_{j i, j k} F_{i}-\rho \mathrm{c}_{S}^{2}\left(G_{j i, j k}-G_{k i, j j}\right) F_{i} \\
+\rho \omega^{2} G_{k i} F_{i}=F_{i}^{(n)},
\end{array}
$$

with summation over repeating indices and with $G_{j i, h k}=$ $\partial_{h} \partial_{k} G_{j i}$ and where $c_{L}$ and $c_{S}$ are the longitudinal and shear wave velocities, respectively, $\rho$ is the volumetric mass density. Further, $F_{i}$ is the discreet force applied at point $x_{0 k} . F_{i}^{(n)}$ is the nonlinear force that depends on the second and third order elastic constants of medium [5]. The nonlinear bulk force

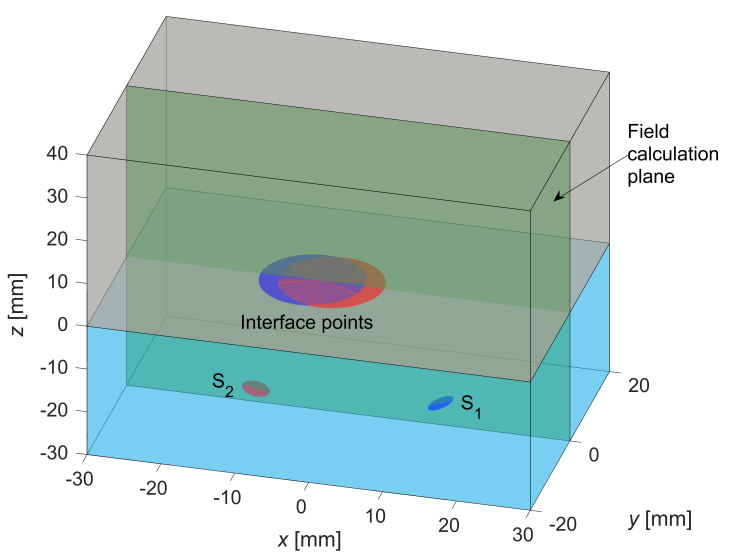

Fig. 1. Problem geometry for the calculation of the nonlinear elastic fields in the elastic medium. Two finite dimension sources $\mathrm{S}_{1,2}$ are submersed in the fluid. 


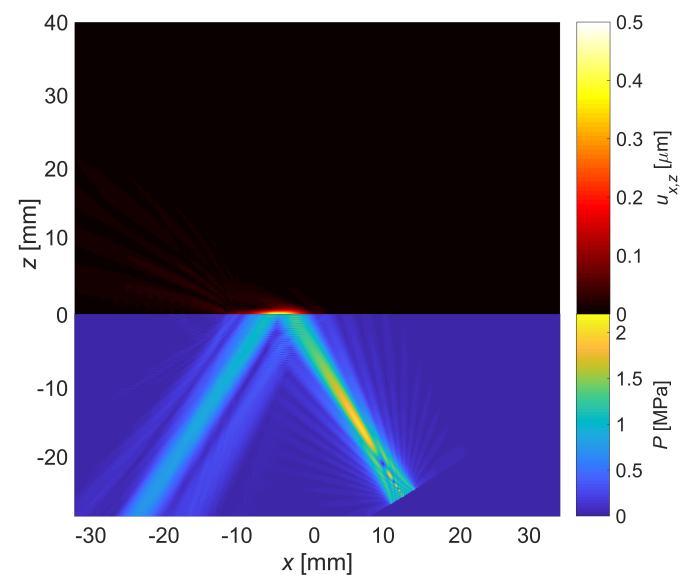

Fig. 2. Linear evanescent ultrasonic fields in the $x 0 z$ plane at $4 \mathrm{MHz}$ : pressure $P$ in the fluid, and $u_{x, z}$ displacements in the solid, of the evanescent longitudinal and shear waves.

can be written in terms of divergence of the nonlinear stress tensor. It was calculated by applying a specific procedure for the interaction interference term as presented in [5], [6]. The two-wave interaction stress tensor $\sigma_{i j}^{(12)}$ for any two interacting displacement wave-fields $\mathbf{u}_{1}$ and $\mathbf{u}_{2}$ was

$$
\sigma_{i j}^{(12)}\left(\mathbf{u}_{\mathbf{1}}, \mathbf{u}_{\mathbf{2}}\right)=\frac{1}{4}\left(\sigma_{i j}\left(\mathbf{u}_{\mathbf{1}}+\mathbf{u}_{\mathbf{2}}\right)-\sigma_{i j}\left(\mathbf{u}_{\mathbf{1}}-\mathbf{u}_{\mathbf{2}}\right)\right)
$$

where $\mathbf{u}(\mathbf{x})=\int \mathbf{G}\left(\mathbf{x}-\mathbf{x}_{0}\right) \cdot \mathbf{F}\left(\mathbf{x}_{0}\right) d^{3} x_{0}$. The divergence of the interference term led to the nonlinear bulk force $F_{i}^{(n)}$.

Using this approach, the following procedure was used to calculate nonlinear elastic fields in submersed elastic isotropic semi-space:

- Formulate the geometry using a grid as outlined in [7]. Note that a full interface between the fluid and solid semispaces is considered for both sources $\mathrm{S}_{1,2}$, see Fig. 1.

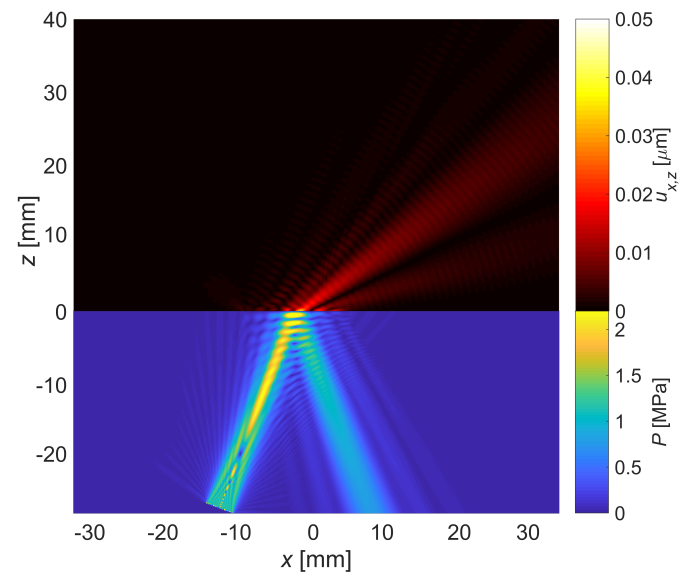

Fig. 3. Linear ultrasonic fields in the $x 0 z$ plane at $6 \mathrm{MHz}$ : pressure $P$ in the fluid, and $u_{x, z}$ displacements in the solid, of propagating shear wave.
Two separate grids are used for each frequency in order to minimise the number of equations to solve.

- Calculate point-source strengths at the interfaces, using global matrices at both initial frequencies [7].

- Calculate the nonlinear forces to propagate nonlinear elastic fields in the solid. In our case, we use the $x 0 z$ plane to visualize the fields, see Fig. 1.

\section{RESULTS}

A numerical model for the nonlinear wave interactions below water-aluminium interface was implemented using the following configuration. Two sources inclined at angles $31.5^{\circ}$ and $20.8^{\circ}$ with diameters of $4 \mathrm{~mm}$ were used to generate initial waves of $4 \mathrm{MHz}$ and $6 \mathrm{MHz}$, respectively. The first source has a vertical offset of $25 \mathrm{~mm}$ from the interface and generated only evanescent fields in the aluminium whilst the second one (vertical offset $27 \mathrm{~mm}$ ) generated a shear wave field in the solid. The horizontal offset was $25 \mathrm{~mm}$ between the sources. Material properties were the same as in [5].

Figs. 2 and 3 show calculated linear ultrasonic fields (pressure in fluid, and $x$ and $z$ displacements in the solid) generated by individual sources at their different initial frequencies. When the incidence beam impinges the interface above the second critical angle, see Fig. 2, we observe a Goos-Hänchen beam shift [8] as well as the evanescent field. Fig. 3 shows the shear wave ultrasonic wave-field in the solid only due to the source angle above the first critical angle.

Fig. 4 shows nonlinear ultrasonic field in the solid generated by the non-classical wave mixing when the interaction occurred between propagating shear wave and evanescent longitudinal and shear waves. It is important to note that the magnitude of the nonlinear field is $75 \mathrm{dBm}$ below that of the linear field in the far-field zone, due to the small interaction volume linked to the small penetration depth of the evanescent wave-field.

Experimental results employing quasi-monochromatic wave excitation of initial waves are presented in [9].

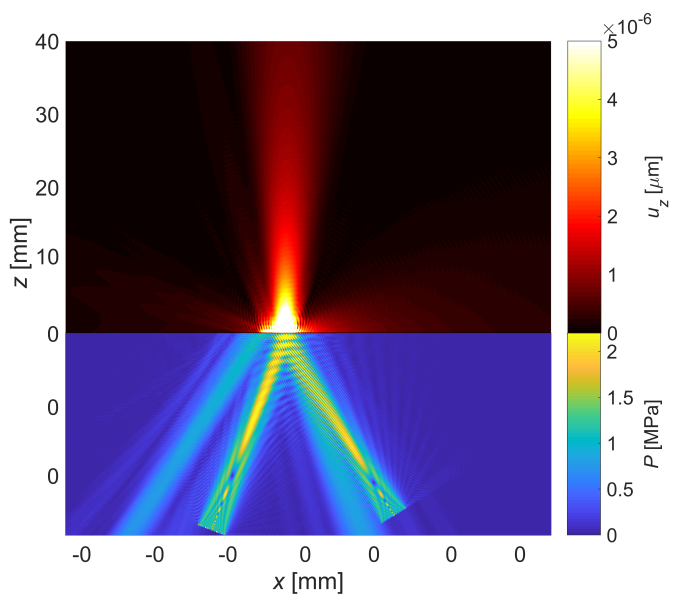

Fig. 4. Nonlinear ultrasonic field in the $x 0 z$ plane: pressure $P$ in the fluid, and $u_{z}$ displacement in the solid, of the nonlinear wave at sum frequency of $10 \mathrm{MHz}$. 


\section{Conclusions}

A 3D frequency-domain Green function method to describe and model nonlinear wave interactions in isotropic hyperelastic media is presented. It is supported by numerical results, calculating the sum frequency due to the ultrasonic response from the mixing at a water-aluminium interface. The analytical nature of the method enables the individual analysis of the nonlinear wave interactions for the different wave combinations $(\mathrm{L}+\mathrm{S}, \mathrm{S}+\mathrm{L}, \mathrm{L}+\mathrm{L}, \mathrm{S}+\mathrm{S})$ with an arbitrary selected wave interaction volume in both the near and far-field zones. The method can be extended to more complex guided wave interactions with localised inhomogeneities in elastic waveguides, by changing boundary conditions.

\section{ACKNOWLEDGMENT}

The authors would like to thank Dr. S. Banerjee from University of South Carolina for his helpful discussions on "Distributed point source method" and for providing a code for the verification of solutions of the linear wave equation.

\section{REFERENCES}

[1] P. Blanloeuil, A. Meziane, and C. Bacon, "2d finite element modeling of the non-collinear mixing method for detection and characterization of closed cracks," NDT E International, vol. 76, pp. 43 - 51, 2015.

[2] M. Hasanian and C. J. Lissenden, "Second order ultrasonic guided wave mutual interactions in plate: Arbitrary angles, internal resonance, and finite interaction region," Journal of Applied Physics, vol. 124, no. 16, p. 164904, 2018.

[3] B. Sarens, B. Verstraeten, C. Glorieux, G. Kalogiannakis, and D. Hemelrijck, "Investigation of contact acoustic nonlinearity in delaminations by shearographic imaging, laser doppler vibrometric scanning and finite difference modeling," Ultrasonics, Ferroelectrics and Frequency Control, IEEE Transactions on, vol. 57, pp. 1383 - 1395, 072010.

[4] V. A. Korneev and A. Demčenko, "Possible second-order nonlinear interactions of plane waves in an elastic solid," Journal of the Acoustical Society of America, vol. 135, no. 2, pp. 591-598, 2014.

[5] M. Mazilu, A. Demčenko, R. Wilson, J. Reboud, and J. M. Cooper, "Breaking the symmetry of momentum conservation using evanescent acoustic fields," Physical Review Letters, vol. 121, p. 244301, 2018.

[6] M. Mazilu, J. Baumgartl, S. Kosmeier, and K. Dholakia, "Optical Eigenmodes; exploiting the quadratic nature of the energy flux and of scattering interactions," Opt. Express, vol. 19, no. 2, pp. 933-945, 2011.

[7] S. Banerjee, T. Kundu, and N. A. Alnuaimi, "DPSM technique for ultrasonic field modelling near fluid-solid interface," Ultrasonics, vol. 46 no. 3, pp. $235-250,2007$.

[8] K. Y. Bliokh and A. Aiello, "Goos-Hänchen and Imbert-Fedorov beam shifts: an overview," Journal of Optics, vol. 15, no. 1, p. 014001, jan 2013.

[9] A. Demčenko, M. Mazilu, R. Wilson, J. Reboud, and J. M. Cooper, "Non-classical second-order nonlinear elastic wave interactions," in IEEE International Ultrasonics Symposium, IUS, vol. 2019-October, 2019. 